\title{
Das Psychomobil
}

\author{
Erhard Taverna
}

Pünktlich werde ich dort abgeholt, wo ich stehe. Wenige Meter neben mir bringt Koni Groher, in weisser Sommerhose und rotem T-Shirt, seine mobile Praxis am Bahnhof Zürich-Stadelhofen zum Stehen. Wir fahren nach Zollikon ins Grüne, während ich es mir neben dem Chauffeur bequem mache. Dem Occasions-Mercedes wurde in Ungarn ein Facelifting verabreicht, neuer Motor, Rostschutz und so weiter, sagt der Fahrer, dafür gehe er hier zum Zahnarzt. Den Möbeltransporter hat er als gelernter Bauführer selber umgebaut und bietet darin seit vier Jahren seine mobile Beratung an. Die Standplätze findet er auf öffentlichen Parkplätzen am See oder, wie heute, an einem schattigen Waldrand, wo er rückwärts, möglichst nahe an einem Bächlein parkt. Aus der früheren Hebebühne wird ein kleiner Balkon mit blauem Spannteppich, den er mit zwei bequemen Klappstühlen möbliert. Wir betreten die geräumige Kabine über eine seitliche Alutreppe und nehmen im Schatten der Buchen Platz. Die Nachmittagssonne beleuchtet durch die Blätter das Innere, wo in einem alten Segeltuchkoffer die ganze Praxisadministration inklusive Musikanlage verstaut ist. In der Wand gegenüber des Eingangs geben vier schmale, hohe Fenster den Blick auf die Strasse frei. Die Spotlampen an der Decke sind für die Sprechstunden

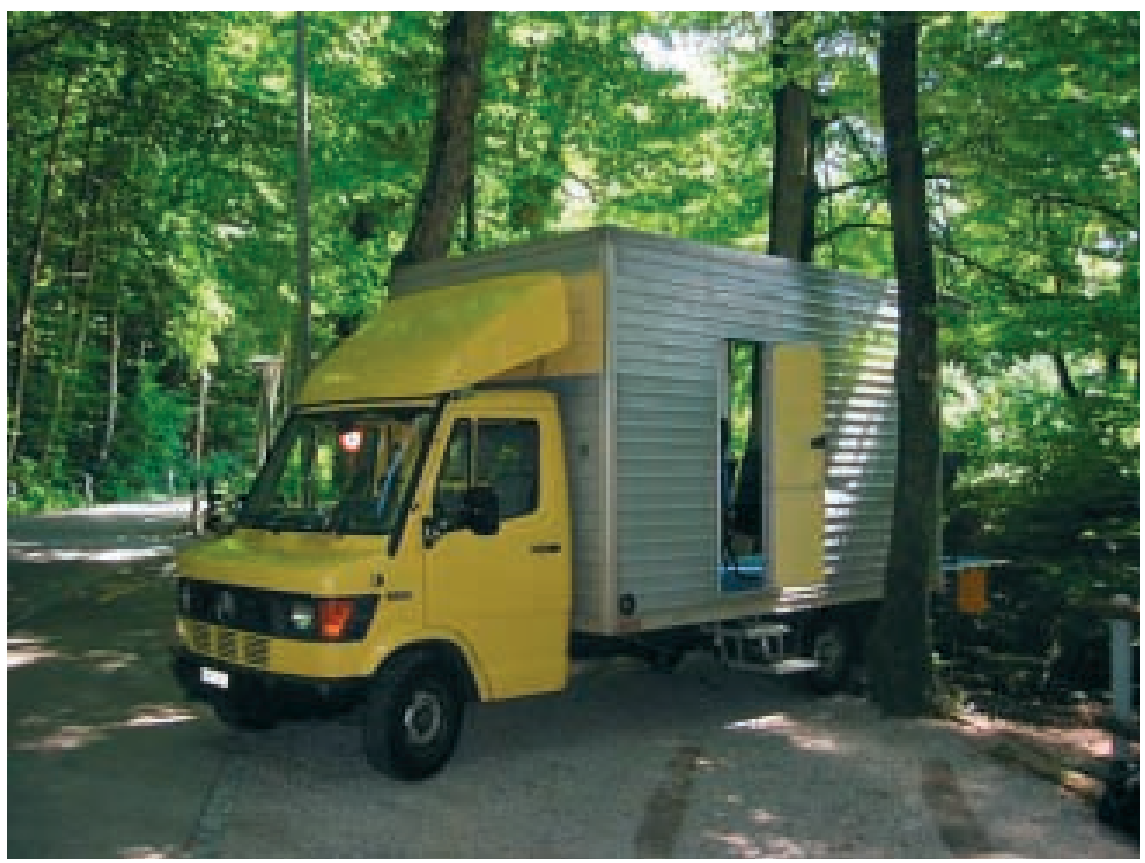

abends und nachts, eine 5-KW-Heizung sorgt auch bei tiefen Aussentemperaturen für frostfreie Beratungsstunden. Je nach Interventionsbedarf wäre ein kleiner Waldspaziergang von hier aus möglich, doch es ist trotz knatterndem Rasenmäher gemütlich und wir bleiben lieber in der kühlen Walddämmerung sitzen.

Über die Blutphobie seiner Ehefrau ist Koni Groher eher zufällig mit der Technik des neurolinguistischen Programmierens NLP bekannt geworden. Damals habe er berufsbegleitend den Grundkurs besucht und dann noch einmal während $2 \times 10$ Tagen den «Master» gemacht und sein Können mit Zusatzkursen in Kommunikation, Hypnose und Provokativer Therapie nach Frank Farrelly erweitert. Stete Mundpropaganda sorgt für neue Kunden, für die er sich als Coach versteht. Sie kommen aus der Agglomeration, aber auch aus Basel, und wenn eine Krise droht, fährt er auch an den Wohnsitz, allerdings dann mit der Bahn. Es kämen Menschen, die etwas verändern wollten, Süchtige, Kinder mit Prüfungsängsten, Bulimiker, auch Paare mit Beziehungsproblemen. «Am liebsten begleite ich Veränderungsprozesse. Ich behandle keine kranken Menschen.» Koni Groher versteht sich als Lebensberater und vermeidet Ausdrücke wie Patient und Therapeut: «Über Bulimie muss ich nichts wissen, mich interessiert, was nachher kommt.» Coaching ist für ihn ein künstlerischer Prozess, den er mit Malen oder Musizieren vergleicht. Er konzentriere sich auf die Eigenkräfte seiner Kunden, mache Konfliktmuster sichtbar und arbeite lösungsorientiert. Praktische Lebenshilfe, vergleichbar mit der Arbeit eines Geburtshelfers. «Es müssen nicht immer Schweiss und Tränen fliessen. Wut, Verzweiflung und Tränen entstehen weniger beim Lösen der Knoten als beim Erkennen, dass da überhaupt solche vorhanden sind.» $\mathrm{Ab}$ und $\mathrm{zu}$ bespricht er sich mit Fachkollegen oder mit guten Bekannten, die medial oder kinesiologisch behandeln.

Der Arbeiter vom Bauamt ist endlich abgezogen, wir baden ungestört im Vogelgezwitscher. Neben seinem Halbtagsjob in seiner Praxis beschäftigt sich Koni Groher intensiv mit Arbeitslosenprojekten für Jugendliche. Wenn er nicht gerade Technik und Beleuchtung für eine Profibühne besorgt oder gleich selber erfolgreich eine Rolle übernimmt als Grossvater, Diener, Graf oder Schlitzohr in Ibsens «Gespenster». 
Sogar das Auto wird anlässlich des 10-JahrJubiläums des PLAYBACK-Theaters in Zürich zur Kleinbühne: hereinspaziert, die eigene Geschichte erzählen und schon geht der Vorhang zum professionellen Playback auf.

Ich gestehe, dass ich ein bisschen neidisch bin. Da hat einer seinen Traum verwirklicht, hängt den bisherigen Job an den berühmten Nagel und macht nur noch, was und soviel er will, ist dank Erbschaft finanziell unabhängig und hat fast unbeschränkt Zeit für die Probleme der - überwiegend weiblichen - Kundschaft. Kein piesackender Berufsverband, keine lästige Auflagen, keine Bewilligungspflicht, praktisch kein Büro und weit und breit keine Krankenkasse. Wechselnde Aussichten, viel Bewegung, grosse Flexibilität und jede Menge Improvisation. Natürlich werden jetzt alle Profis der
Therapeutenszene mit Recht die Stirne runzeln. Ja, der Mann verstösst gegen viele Berufsregeln, er ist sehr bescheiden ausgebildet und er mag einem gelegentlich vorkommen wie der bekannte Reiter auf dem Weg über den Bodensee.

Was mich an ihm beeindruckt hat, ist seine innere Ruhe und spürbare Reife. Er hat das, was kein Fähigkeitszeugnis und kein Spezialarzttitel garantieren können. Auf ihn als engagierten Helfer trifft zu, was der von ihm verehrte Autor Kurt Götz über den Humor aussagt: «Neben Geist und Witz setzt er vor allem ein grosses Mass an Herzensgüte voraus. Von Geduld, Nachsicht und Menschenliebe.»

Koni Groher, Rebweg 24, 8700 Küsnacht www.konigroher.ch

\section{Santé publique et tabagisme}

\section{De l'aveuglement historique à la nécessité contemporaine d'une éthique de la responsabilité*}

\section{J. Aubert}

\footnotetext{
* En prolongement de la journée mondiale sans tabac proposée par l'OMS le 31 mai 2002.
}

Correspondance:

Dr Jacques Aubert

13 , rue de Soleure

CH-2525 Le Landeron

Tél. 0327512326

Fax 0327516413

e-mail: jac.aubert@net2000.ch

\section{Introduction}

La fumée du tabac constitue aujourd'hui un fléau menaçant dramatiquement la santé de la population. Le tribut que les fumeurs paient au plaisir de la cigarette est très lourd puisque deux sur quatre en meurent, et qu'un quart des fumeurs décèdent prématurément (entre 40 et 65 ans). L'OMS estime que le nombre de morts prématurées liées au tabagisme va passer de 4 millions en 1998 à 10 millions en 2030 ...

Il faut agir. Or, les efforts de prévention primaire auprès des jeunes restent un échec: les jeunes sont de plus en plus nombreux à fumer, et toujours plus tôt. Faut-il s'en étonner? Pas vraiment, comme nous allons le voir. Faut-il baisser les bras? Sûrement pas. Mais favoriser l'action juste suppose de nourrir celle-ci de substantielles considérations historiques et scientifiques, et aussi de l'abreuver de quelques réflexions de philosophie politique. Je voudrais, modestement, y contribuer ici.

\section{De l'aveuglement et des plaisirs innocents de Catherine de Médicis...}

Pendant des siècles, l'homme a vécu sans tabac; on se délectait au théâtre sous Périclès ou aux jeux du cirque sous Néron sans pause cigarette. L'usage du tabac remonte à Christophe Colomb en 1492. En 1518, les premières graines de tabac ramenées en Europe sont cultivées au Portugal et en Espagne. L'ambassadeur de France au Portugal, Jean Nicot diffuse le tabac en Europe. En bon courtisan, il cherche à se gagner les faveurs de la redoutable Catherine de Médicis en lui envoyant des graines de tabac réputées capables de soulager les migraines atroces dont souffrait la reine.

La première vente de cigarettes est réalisée en 1843. La coutume du «fumoir» stigmatise l'un des premiers effets de la consommation de cigarettes en société: on ne savait rien des effets nocifs de la fumée sur la santé des non-fumeurs, mais on avait des égards pour les non-fumeuses. 
Dans les pays développés, la première moitié du $20^{e}$ siècle connaît l'expansion du tabagisme masculin, la deuxième celle du tabagisme féminin, symbole d'émancipation. Si bien que le $20^{\mathrm{e}}$ siècle passera à la postérité comme le «siècle des cigarettes».

Très tôt, les financiers voient la bonne affaire: taxer une drogue comme le tabac est parfaitement rémunérateur pour qui encaisse l'impôt. Taxer le tabac, favoriser sa production et son commerce, c'est assurer à l'Etat et à l'industrie une source intarissable de revenus. Richelieu le comprend, qui, dès 1629 taxe le tabac à l'importation. En 1674, Colbert en fait un monopole d'Etat. Necker écrit en 1784: «L'impôt sur le tabac est de toutes les contributions la plus douce et la plus imperceptible et on la range avec raison dans la classe des habiles inventions fiscales».

\section{Et des données modernes de la connaissance médicale ...}

Catherine de Médicis appréciait «l'herbe à Nicot» pour se détendre et calmer ses migraines; elle était bien innocente, trouvant à cet usage quelque agrément, ignorant tout des effets potentiellement délétères de cette consommation sur la santé. «Le siècle des cigarettes» va nous permettre, à nous les médecins, de cumuler les données épidémiologiques et scientifiques de l'extrême nocivité du tabac pour la santé.

En 1962 est publié à Londres le $1^{\text {er }}$ rapport médical faisant état des risques de la cigarette pour la santé: «la fumée de la cigarette est une cause importante des cancers du poumon». Puis les rapports montrant les effets désastreux de l'exposition active à la fumée se succèdent. On découvre le phénomène du tabagisme passif: l'exposition passive à la fumée augmente pour les non-fumeurs le risque d'infection, de cancer et même d'ischémie cardiaque. Enfin, on observe une ascension vertigineuse de l'incidence du cancer du poumon chez les femmes, parallèlement à l'augmentation du nombre de fumeuses (le nombre de femmes atteintes d'un cancer du poumon a augmenté de $30 \%$ au cours des sept dernières années).

Les constatations sont sans appel: la fumée est la première cause de mort en Occident, loin derrière les accidents de la route (le tabac tue treize fois plus que les accidents de la route). En Suisse, la majorité des décès prématurés entre 35 et 70 ans sont dus à la fumée, par cancer ou infarctus.

L'épidémie occidentale se développe en pandémie mondiale.

\section{... aux tartufferies contemporaines}

En dépit des révélations scientifiques successives, les industriels du tabac ont continué de nier que fumer provoque des cancers et que la nicotine crée une dépendance. A la fin du $20^{\mathrm{e}}$ siècle, les contributions scientifiques ébranlent enfin les pseudo-certitudes et la pseudo-innocence des cigarettiers: en 1997, le fabricant de Chesterfield aux USA reconnaît avoir menti et transgressé plusieurs lois: les industriels connaissaient depuis longtemps les méfaits du tabac. Cet industriel note dès lors sur ses produits: «cigarette is addictive».

Allons-nous longtemps encore jouer les Tartuffes?

Jusqu'à quand sera acceptée l'hypocrisie de l'industrie du tabac, qui s'enrichit copieusement en prenant les jeunes pour cible et en recourant au slogan pervers des cigarettes dites légères, lesquelles présentent surtout l'avantage d'éviter la nausée des premières bouffées et par conséquent de mieux crocher le jeune client?

Jusqu'à quand sera acceptée l'hypocrisie de la Suisse, qui se constitue paradis fiscal pour les cigarettiers (57\% des parts fiscales en Suisse contre $72 \%$ en Italie, $75 \%$ en France, $85 \%$ au Danemark) et cautionne ainsi la production de cigarettes pour, notamment, l'exportation dans les pays du Tiers-Monde où on exporte sciemment une épidémie?

Jusqu'à quand sera acceptée l'hypocrisie d'une société qui se réjouit de garnir ses caisses cantonales et fédérales avec les revenus de la vente du tabac?

Le 5 février 2002 neuf organisations de santé (dont la FMH) et de défense des consommateurs ont proposé à Berne une augmentation du prix des cigarettes (de Fr. 4.80 à 5.60) et la création d'un fonds de prévention du tabagisme. Il a en effet été démontré qu'une augmentation de 10\% du prix des cigarettes diminue la consommation de $4 \%$ dans la population générale et de $8 \%$ chez les 16-17 ans.

Le 20 février 2002 le Conseil fédéral rend sa décision: il refuse aussi bien les modifications de l'impôt sur le tabac que la création d'un fonds de prévention, s'alignant ainsi, contre la position de la FMH, sur la position ambiguë et même paradoxale de certains médecins comme Barbara Polla, députée à Berne ... Mais il maintient un fonds de 20 millions par an pour la subvention de la culture du tabac.

Ces refus politiques du gouvernement s'inscrivent dans un contexte où la publicité pour le tabac reste autorisée et, à l'évidence, performante et juteuse. On rappellera que les cigaret- 
tiers suisses dépensent 120 millions par an pour leur publicité, alors que le budget annuel de la Confédération pour la prévention du tabagisme est de 6 millions ... «La société de consommation, par son matraquage de signaux, place l'adolescent en perpétuelle tentation» relève la sociologue Monique Dagnaud [1].

L'épidémie croissante et préoccupante du tabagisme chez les jeunes en Suisse n'est pas tant la conséquence d'un échec de la prévention; elle est à lire surtout comme un formidable succès de la promotion du tabagisme menée dans ce pays.

Allons-nous longtemps encore jouer les Tartuffes?

Une éthique s'impose.

\section{Du principe de responsabilité au principe de précaution}

Le philosophe Hans Jonas développe dans «Le principe responsabilité» [2] le concept philosophique de la nécessité, pour les sociétés technologiques, d'une conversion à la mesure et à la précaution. C'est une précaution fondée sur la responsabilité. En l'occurrence, la responsabilité suppose l'écoute attentive des données de la science médicale et le développement subséquent, par les collectivités, d'actions préventives de type principe de précaution qui soient en quelque sorte la matérialisation du principe de responsabilité, sa mise en forme.

Dans les faits, le principe de précaution peine à trouver une traduction politique: la publicité pour le tabac continue d'inonder les espaces où se regroupent les jeunes (gares, stations de bus, cinémas) et la Cour européenne a même annulé la directive européenne interdisant la publicité pour le tabac ...

La Suisse, pourra-t-elle longtemps encore, en toute impunité, continuer de favoriser cette «industrie de la mort», et lui garantir ad libitum ses espaces publicitaires? Sans risquer de s'exposer à l'avenir à des demandes de dommages et intérêts de la part de familles de victimes, et peutêtre un jour de la part d'autres Etats? Un procès a déjà été intenté en France à un chef d'entreprise par une femme dont la sœur, non-fumeuse, est décédée à 45 ans d'un cancer du poumon, pour avoir pendant 15 ans, respiré la fumée de ses collègues de travail. Un Tribunal Lyonnais a condamné la SNCF pour n'avoir pas sanctionné la présence de fumeurs dans une gare ...
Aujourd'hui l'équivalent pour le pénal du non-respect du principe de précaution, c'est l'introduction de nouveaux crimes. En effet, comme on a désormais accepté, dans l'espace public, le concept du "principe de précaution», on a introduit, dans le pénal, son équivalent, à savoir la «mise en danger de la vie d'autrui».

L'insouciance et l'inconséquence avec lesquelles les sociétés occidentales gèrent la problématique du tabagisme constituent un terrain sociologique favorisant la judiciarisation du monde. Cette judiciarisation du monde n'est rien d'autre que la conséquence de notre incapacité de gérer le principe de précaution là où il devrait l'être, c'est-à-dire dans la politique.

Il n'est pas de question politique, aux EtatsUnis, écrivait Tocqueville, qui ne se résolve tôt ou tard en question judiciaire. Si la politique ne reprend pas ses droits, il est fort à craindre que le tribunal devienne, en Europe aussi, un des lieux névralgiques de la démocratie. Une telle irruption du droit dans la vie publique serait évidemment fort regrettable. Mais c'est bien parce qu'il y a absence ou démission du politique que sont confiées aux juges des tâches qui ne leur reviennent pas.

En matière de santé publique, à nous les médecins, d'effectuer régulièrement des injections de rappel auprès de nos responsables politiques pour leur signifier que le tabagisme tue beaucoup plus d'êtres humains, souvent encore jeunes, que le SIDA, l'héroïne ou l'automobile, et qu'il faut agir. Je ne crois pas à la force intrinsèque de la logique marchande, si nous sommes déterminés. Un seul gardien du temple peut faire filer des squattes de marchands du temple. Si nous pleurnichons parce que la logique marchande nous déborde, c'est que nous n'existons pas et que nous n'avons plus que les larmes pour pleurer.

\section{Références}

1 Dagnaud M. Les enfants, acteurs courtisés de l'économie marchande. Rapport à Jack Lang, ministre de l'éducation nationale. 18 février 2002.

2 Jonas H. Le principe responsabilité. Une éthique pour la civilisation technologique. Paris: Flammarion; 1998 